\title{
HERNIA INGUINAL RECIDIVADA. TRATAMIENTO AMBULATORIO CON ANESTESIA LOCAL POR LA VÍA ABIERTA ANTERIOR*
}

\author{
Drs. Alberto Acevedo F. ${ }^{1,2}$, Jorge León S. ${ }^{1,2}$, Gabriel García P. ${ }^{1,2}$ \\ 1 Unidad de Hernias del Centro de Referencia de Salud (CRS) del Servicio de Salud Metropolitano Oriente \\ (SSMO). \\ 2 Departamento de Cirugía del Campus Oriente de la Facultad de Medicina de la Universidad de Chile. \\ Santiago, Chile.
}

\begin{abstract}
\section{Ambulatory repair of recurrent hernias. Experience in 70 cases}

Background: Recurrence is the most common long term complication of inguinal hernia repair. Aim: To report the experience of ambulatory treatment of hernia recurrence. Patients and Methods: Seventy patients aged $56 \pm 14$ years (59 men), with a low surgical risk and a body mass index below $40 \mathrm{~kg} /$ $\mathrm{m}^{2}$ were admitted to the program. The surgical repair of the hernia was performed under local anesthesia on an ambulatory basis. All patients received antibiotic and prophylaxis for venous thrombosis. Results: The surgical procedure lasted $54 \pm 25$ minutes. A tissue repair was performed in 12 cases and a prosthetic one in 48. The ductus deferens was accidentally sectioned in one case. The postoperative complications were one hematoma which did not require surgery and two superficial infections that healed in an ambulatory basis. A long term follow up, for $9.3 \pm 1.9$ years after surgery was possible in $79 \%$ of cases. Two recurrences $(3.5 \%)$ were observed and one patient complained of a light, intermittent pain in the inguinal region. There were two recurrences after tissue repair, both occurring after a Lichtenstein repair (4.2\%). Ninety two percent of patients were satisfied or very satisfied with the procedure. Conclusion: Ambulatory treatment of recurrent hernias is feasible and safe.
\end{abstract}

Key words: Hernia, recurrence, ambulatory surgery.

\section{Resumen}

Objetivos: La recidiva continúa siendo la más frecuente complicación tardía de las herniorrafias inguinales primarias; estas tasas son aún mayores cuando se actúa sobre las hernias recidivadas. En el presente estudio prospectivo, observacional, descriptivo, damos cuenta de nuestros resultados en una serie de pacientes intervenidos en forma electiva, en el Centro de Hernias del CRS Cordillera, entre los años 1998 y 2009 por

\footnotetext{
Los autores no refieren conflictos de interés.

Correspondencia: Dr. Alberto Acevedo F. aacevedof@gmail.com
}

*Recibido el 30 de enero de 2014 y aceptado para publicación el 19 de enero de 2015. 
hernias inguinales recidivadas. Material y Métodos: La cirugía se efectuó, con anestesia local asistida, en forma ambulatoria, tras una adecuada selección de los pacientes y firma de un consentimiento informado. Se intervinieron 70 pacientes, 59 varones y 11 mujeres con una edad promedio de 56,2 $\pm 13,9$ años. Se usó profilaxis antibiótica y de la trombosis venosa profunda. Resultados: La duración promedio de la cirugía fue de $54 \pm 25,3$ min. Se efectuó reparación tisular en 12 y protésica en 48 casos. El conducto deferente se seccionó en forma accidental en un caso. En el postoperatorio hubo un hematoma y 2 infecciones superficiales que se trataron ambulatoriamente. El seguimiento alejado a un promedio de 9 (4-14) años se efectuó en el $79 \%$ de la serie, detectándose 2 recidivas $(3,5 \%)$ y un caso de inguinodinia leve. Las 2 recurrencias se apreciaron en las reparaciones con técnica de Lichtenstein (4,2\%). El 92\% de los enfermos refirió estar satisfecho o muy satisfecho con el procedimiento. Conclusiones: La reparación ambulatoria con anestesia local, en manos expertas, es una alternativa a ser considerada en el tratamiento de las hernias inguinales recidivadas.

Palabras clave: Hernia inguinal recidivada, anestesia local, cirugía ambulatoria, herniorrafia tisular, Lichtenstein.

\section{Introducción}

La recurrencia es la complicación más frecuente a largo plazo en herniorrafia inguinal. La especialización en cirugía herniaria, y el desarrollo de centros especializados en esta cirugía ha permitido bajar estas tasas, con técnicas fasciales (tisulares), a cifras cercanas al $1 \%{ }^{1,2}$. Numerosos autores dan cuenta de recidivas del orden del $1 \%$ con el uso de la técnica de Shouldice 3 . En manos del cirujano general, sin embargo, las tasas de recidivas utilizando estas técnicas han permanecido alrededor del $10 \%{ }^{4-6}$. La introducción de las técnicas "tension-free" utilizando mallas y de la cirugía laparoscópica de las hernias ha contribuido a bajar estas tasas, pero, a pesar de estos adelantos, las hernias inguinales recidivadas constituyen aún una elevada proporción de las herniorrafias inguinales, con escasa tendencia a disminuir ${ }^{6,7}$.

La reparación de las hernias recidivadas puede hacerse abierta por vía anterior, a través de la antigua cicatriz, utilizando una prótesis o una técnica fascial, o por vía preperitoneal (posterior), instalando una malla, usando un acceso abierto o uno laparoscópico. La vía anterior y la anestesia local son usadas, especialmente en centros dedicados a la cirugía herniaria ${ }^{8,9}$.

En el presente estudio damos cuenta de nuestra experiencia con el tratamiento de las hernias inguinales recidivadas intervenidas con anestesia local utilizando la vía abierta anterior, en un centro ambulatorio especializado en el tratamiento de las hernias de la pared abdominal.

\section{Material y Método}

El presente es un estudio prospectivo observacional descriptivo realizado en los pacientes con hernia inguinal recidivada, efectuado en la Unidad de Cirugía Mayor Ambulatoria (CMA) del Centro de Referencia de Salud (CRS) Cordillera Oriente, entre los años 1998 y 2009. Este Centro presta atención especializada a los pacientes derivados desde los consultorios de las comunas de Peñalolén y de Macul del Servicio de Salud Metropolitano Oriente, portadores de hernias abdominales electivas. Los pacientes en condiciones físicas y psicosociales compatibles con la cirugía ambulatoria con anestesia local son intervenidos en este Centro ${ }^{10,11}$.

Se incorporaron al estudio pacientes ASA 1 y 2, con IMC de hasta 40. La diabetes mellitus compensada y la hipertensión arterial adecuadamente tratada no fueron una causa de exclusión. La Tabla 1 da cuenta de las características de los pacientes de esta serie clínica.

Tras el examen de ingreso y realizados los exámenes e interconsultas necesarias en cada caso, los pacientes firmaron un consentimiento informado. El día de la intervención concurrieron en ayunas de sólidos por $12 \mathrm{~h}$ y $\sin$ suspender sus medicamentos habituales lo que es especialmente trascendente en el caso de los pacientes diabéticos e hipertensos.

Una vez preparada y aseada la piel se instaló un suero de mantención y se administró profilaxis antibiótica con Cefazolina ${ }^{\circledR} 1 \mathrm{~g}$ intravenosa. La profilaxis tromboembólica se efectuó mediante vendaje elástico de las piernas y deambulación post operatoria precoz. Anestesia local con sedación. Se instaló un suero fisiológico y se procedió a administrar la sedación consistente rutinariamente en Midazolam ${ }^{\circledR}$ $5 \mathrm{mg}$ y Amidona ${ }^{\circledR} 10 \mathrm{mg}$. La Amidona, diluida en $10 \mathrm{ml}$ de suero fisiológico se administró en bolos de $2 \mathrm{ml}$ según demanda. La infiltración anestésica se realizó con 160 cc de Lidocaína al 0,5\%, alcalinizada y adicionada con $0,25 \mathrm{mg}$ de Adrenalina. En caso de obesidad se aumentó el volumen a $200 \mathrm{cc}$ al $0,4 \%$. Se empleó la técnica de infiltración por planos (epidermis, dermis, tejido celular subcutáneo, plano bajo la aponeurosis del músculo oblicuo interno (AMOI), plano muscular y plano preperitoneal) ${ }^{11}$. 
Tabla 1. Características de los pacientes con hernia inguinal recidivada

\begin{tabular}{|c|c|}
\hline \multicolumn{2}{|l|}{ Característica } \\
\hline $\operatorname{IMC}(\bar{x} \pm s t)$ & $26 \pm 4,5$ \\
\hline Patología asociada (n) & 29 \\
\hline Diabetes mellitus & 5 \\
\hline HTA & 18 \\
\hline LCFA & 2 \\
\hline Epilepsia & 1 \\
\hline Cardiopatía & 3 \\
\hline Antiguedad de la recidiva $(\overline{\mathrm{x}} \pm \mathrm{st})$ & $5,9 \pm 7,4$ \\
\hline $\begin{array}{l}\text { Tamaño de la hernia (n) } \\
>10 \mathrm{~cm} \\
5-10 \mathrm{~cm} \\
<5 \mathrm{~cm}\end{array}$ & $\begin{array}{r}8 \\
24 \\
36\end{array}$ \\
\hline Inguinoescrotal (n) & 9 \\
\hline Lado derecho (n) & 45 \\
\hline Lado izquierdo (n) & 23 \\
\hline Bilateral (n) & 2 \\
\hline Hernia por deslizamiento (n) & 1 \\
\hline
\end{tabular}

Considerando las adherencias y fibrosis interplanos presentes en las hernias recidivadas se efectuaron múltiples infiltraciones en cada plano. El técnico paramédico llevó el control de los signos vitales, administró los fármacos tras indicación del anestesista y llenó la ficha anestésica.

\section{Técnica quirúrgica}

Se procedió en forma diferente según la operación anterior hubiera sido efectuada con prótesis (10 casos) o con técnicas fasciales (60 casos).

\section{Técnica previa fascial}

Tras resección de la cicatriz se incidió el plano subcutáneo para exponer la AMOI, comprobando la posición normal o desplazada al tejido celular subcutáneo del cordón espermático. Incidida la AMOI se controló el cordón y se disecó y trató el o los sacos herniarios. Tras identificar las estructuras anatómicas y evaluarlas, se decidió la cirugía reconstructiva a realizar. La técnica fascial se utilizó en aquellos casos en que la pared posterior del canal inguinal (PPCI) aparecía intacta, intocada por la cirugía anterior. Consistió en suturar el arco del músculo transverso al tracto iliopubiano (Madden) o al ligamento inguinal (Bassini), mediante puntos separados de Prolene 2-0. También se usó la técnica de
Shouldice. Cuando la fibrosis cicatricial dificultaba el acercamiento de los planos de sutura efectuamos una maniobra de relajación del músculo recto ${ }^{12}$. La técnica protésica de Lichtenstein se utilizó en aquellos casos en que se comprobó deterioro de las estructuras anatómicas y consistió en la instalación de una placa de malla de Prolene según técnica descrita por $\mathrm{Amid}^{13}$.

Técnica previa con malla: Manejo de piel y tejido celular subcutáneo similar al anterior. La disección se centró en el saco herniario, protegiendo el cordón espermático. En ningún caso se procuró resecar la malla. Se disecó el saco hasta exponer el anillo herniario identificando sus bordes aponeuróticos así como el borde desprendido de la malla, resuturándola o instalando un tapón de Gilbert. El tapón se fijó a las estructuras firmes del anillo, incluyendo la malla previamente instalada.

Una vez completada la cirugía el paciente se sentó en la mesa quirúrgica, se incorporó y deambuló hasta la sala de recuperación vecina, acompañado por el personal de anestesia, donde estuvo en observación por dos horas hasta ser dado de alta una vez comprobada la tolerancia a la deambulación y la emisión de orina. Los pacientes se trasladaron a su domicilio por sus propios medios. Se recomendó a los pacientes evitar el reposo en cama y asumir a la brevedad sus actividades habituales en el hogar. Como analgesia posoperatoria se indicó Ibuprofeno® $400 \mathrm{mg}$ y Paracetamol ${ }^{\circledR} 500 \mathrm{mg}$ cada $8 \mathrm{~h}$.

\section{Seguimiento postoperatorio}

Se efectuó a las $24 \mathrm{~h}$, a la semana y al mes de la intervención. En cada oportunidad se llenó una ficha pre impresa conteniendo la evaluación del dolor intra y postoperatorio mediante EVA de 10 puntos, la actividad en el hogar y la presencia de manifestaciones adversas (náuseas, vómitos, cefalea, mareos, dificultades para obrar u orinar), la necesidad de realizar una consulta de urgencia o una re hospitalización. Se consignó la presencia de fluxión funículotesticular, de hematoma e infección de la herida.

\section{Seguimiento a largo plazo}

Se realizó mediante una encuesta telefónica. Se pesquisaron las recidivas y la presencia de Inguinodinia. Ésta se evaluó mediante una escala analógica de 5 puntos y estableciendo si el dolor producía limitación funcional. Cuando hubo alguna duda respecto a la presencia de una recidiva herniaria se efectuó un examen presencial por un cirujano del equipo. La satisfacción con el procedimiento se investigó mediante una escala analógica de 4 puntos (muy insatisfecho, insatisfecho, satisfecho, muy satisfecho).

La información de los pacientes se introdujo en 
una base de datos confeccionada con el software de cálculo epidemiológico Epi Info que permite realizar la estadística descriptiva de los datos.

\section{Resultados}

En el presente estudio se da cuenta de 70 pacientes, 59 varones y 11 mujeres con una edad promedio de 56,2 $\pm 13,9$ años. En 51 ocasiones se trató de una primera recidiva, en 6 de una segunda, en 3 de una tercera y en 1 de una cuarta recidiva. La operación anterior había sido fascial en 59 oportunidades y protésica en 11 (Tabla 1).

La duración del acto quirúrgico fue en promedio de $54 \pm 25,3 \mathrm{~min}$ y el dolor durante la cirugía fue calificado con un EVA de 2,3 $\pm 1,6$. Cinco pacientes refirieron una EVA mayor a 4. La cirugía se completó con anestesia local en todos los casos. No se produjeron manifestaciones adversas de gravedad durante el procedimiento.

En los 59 pacientes con cirugía tisular previa, apreciamos que el cordón espermático se encontraba desplazado al tejido celular subcutáneo en 13 oportunidades y en su sitio anatómico, bajo la AMOI en el resto.

En 12 pacientes con un defecto de tipo 2 ó 3 según la clasificación de Gilbert ${ }^{13}$, en los cuales la PPCI aparecía indemne y de buena calidad, efectuamos una reparación tisular y en 48 con defectos tipo 4 o cuando se apreció una calidad deficiente de las estructuras anatómicas utilizamos la técnica de Lichtenstein (Tabla 2). La indemnidad de la PPCI en un número elevado de pacientes sólo es explicable aceptando que el cirujano no efectuó técnica alguna de reparación y ello debe ser atribuido a una grave falta de formación quirúrgica.

En los 11 pacientes con una cirugía previa protésica dirigimos la disección hacia el saco separándolo del cordón espermático y exponiendo el anillo herniario. Tras liberar el saco de sus adherencias

Tabla 2. Operación previa y técnica usada en la reparación de los pacientes de esta serie

\begin{tabular}{|lclr|}
\hline $\begin{array}{l}\text { Operación } \\
\text { previa }\end{array}$ & n & $\begin{array}{l}\text { Técnica de } \\
\text { reparación }\end{array}$ & n \\
Lichtenstein & 11 & Tapón de Gilbert & 10 \\
Fascial & & Re sutura & 1 \\
& 59 & Bassini & 4 \\
& & Shouldice & 6 \\
& & Madden & 2 \\
Total & 70 & Lichtenstein & 47 \\
\hline
\end{tabular}

al anillo lo redujimos al espacio pre peritoneal y colocamos un tapón construido con la técnica de Gilbert $^{13}$, que se fijó a las estructuras vecinas. En un paciente fue posible re suturar el borde desprendido de la malla a los tejidos vecinos y al tubérculo pubiano. En ningún caso extirpamos la malla instalada en la cirugía anterior. La posición de las recidivas fue en la vecindad del tubérculo pubiano en 10 casos y junto al anillo inguinal profundo en uno (Tabla 2).

Hubo 2 complicaciones operatorias con sección del conducto deferente, una de necesidad por adherencias de éste al anillo herniario (involucramiento en un punto de sutura) y otra accidental. Ambos eran casos de recidiva múltiple.

Los controles planificados se efectuaron en todos los pacientes. El dolor fue el síntoma predominante en el primer control, a las $24 \mathrm{~h}$, con un EVA promedio de 4,5 $\pm 1,96$, que descendió a $3,1 \pm 1,4$ a la semana y a $2,2 \pm 0,96$ al mes de la intervención. Seis pacientes presentaron náuseas el primer día de la intervención. No hubo casos de retención de orina y ningún paciente requirió consultar a una unidad de emergencia o debió ser re hospitalizado.

En el postoperatorio hubo un hematoma que no requirió de intervención quirúrgica y 2 casos de infección superficial de la herida. Ambos se trataron exitosamente en forma ambulatoria. Siete pacientes evolucionaron con fluxión funículo-testicular ${ }^{14}$ que fue severa en 2 oportunidades (diámetro del cordón espermático $>3 \mathrm{~cm}$ ). Todas ellas evolucionaron en forma favorable. No hubo casos de isquemia testicular.

El seguimiento alejado efectuado en promedio a los 9 (4-14) años después de la intervención fue posible en 57 oportunidades ( $79 \%$ de los casos). Dos pacientes habían fallecido y 10 fueron inubicables. La recidiva general se apreció en 2 pacientes $(3,5 \%)$. Ésta se apreció en los pacientes intervenidos con la técnica de Lichtenstein $(4,2 \%)$. No hubo recidiva en los 12 pacientes intervenidos con técnicas fasciales ni en los 10 en los que se instaló un tapón de Gilbert. Sólo un paciente presentó una Inguinodinia de carácter intermitente y moderado que no le impedía realizar sus actividades habituales. El 92\% de los pacientes entrevistados refirió estar satisfecho o muy satisfecho con el procedimiento utilizado.

\section{Discusión}

Los estudios realizados en las bases de datos danesas $^{16}$, suecas ${ }^{17}$ y alemanas ${ }^{6}$ de las herniorrafias inguinales dan cuenta de un 11 a $14 \%$ de hernias recidivadas en el total de hernias inguinales intervenidas cada año. Estas cifras se mantuvieron en los años que duraron estos estudios o descendieron 
sólo levemente, a pesar del uso de mallas en la reparación herniaria. En el Centro de Hernias del CRS Cordillera las herniorrafias en hernias inguinales recidivadas constituyeron aproximadamente el $6 \%$ de los pacientes intervenidos cada año en el período que abarca este estudio.

Bisgaard ${ }^{29}$, en estudios efectuados en la Danish Hernia Database, destaca que las técnicas fasciales siguen siendo utilizadas en las hernias inguinales recidivadas por los cirujanos generales, pero da cuenta también de una tasa de re operaciones (re recurrencias) promedio del $6,7 \%$ con un seguimiento de 30 meses. Haapaniemi ${ }^{33}$, basado en el Swedish Hernia Register, informa de 4,6\% de reoperaciones en hernias recidivadas con un seguimiento de 2 años.

Si bien el objetivo de este estudio es destacar la factibilidad de la reparación de las hernias recidivadas en forma ambulatoria con anestesia local, es necesario mencionar que no se detectaron recidivas en los pacientes tratados con técnicas fasciales. Esta situación es extremadamente rara pues las publicaciones que dan cuenta del uso de estas técnicas dan cuenta de una elevada tasa de recidivas. Janu ${ }^{31}$, reporta $18 \%$ de re recurrencias tras el uso de técnicas fasciales y Ljzermans ${ }^{34}$, del $23 \%$ a 3 años, y por ello es necesario destacar acá las características de la técnica fascial que hemos utilizado.

La técnica fascial que describimos se diferencia del Bassini clásico por cuanto no incidimos la fascia transversal (PPCI) sino que exponemos el borde de la aponeurosis del músculo transverso mediante una maniobra que hemos llamado "tensión del arco del transverso". Ella expone en forma neta el borde aponeurótico y hace posible efectuar una sutura perfectamente controlada y con un mínimo de tensión. En 1992 dimos a conocer esta técnica en una serie clínica de 20 pacientes intervenidos por una hernia recidivada en los que no apreciamos recurrencias con un seguimiento a 5 años ${ }^{12}$.

En el presente estudio utilizamos la misma técnica en la reconstitución de la PPCI, suturando el arco del músculo transverso al ligamento inguinal (Bassini) o al tracto iliopubiano (Madden) o realizando una técnica de Shouldice, no apreciando recurrencias tras un seguimiento promedio de 9 años.

Nuestras observaciones sobre la indemnidad de la PPCI en algunos pacientes que hemos intervenidos por hernias recidivadas coinciden con las realizadas por Moschabeck ${ }^{6}$. En el $90 \%$ de los casos intervenidos por ella, en los cuales se habría realizado un Bassini o un Shouldice en la primera operación, la PPCI (fascia transversal) estaba intacta poniendo en evidencia una seria falla técnica. En 2/3 de estos pacientes la calidad de los tejidos permitió reparar la recidiva herniaria con técnica fascial.

En 47 sujetos del presente estudio, el grado de distorsión anatómica, la existencia de fibrosis cicatricial y el tamaño del defecto, hicieron necesaria la interposición de una malla con la técnica de Lichtenstein. No es de extrañar que, por estas condiciones, en este grupo, hayamos tenidos las únicas recurrencias de este estudio.

El uso de la técnica de Lichtenstein en el tratamiento de las hernias primarias ha logrado disminuir las recidivas a niveles entre el 1 y el 5\%. Estas tasas son el resultado de estudios en pacientes electivos, con seguimientos variables, provenientes de centros individuales o de estudios multicéntricos ${ }^{16-27}$. En las hernias recurrentes se ha reducido también la tasa de recidivas, pero estas persisten en niveles del 3,8 al $15,8 \%{ }^{26-34}$. Los resultados de nuestra serie con una recidiva del $3,5 \%$ con un seguimiento promedio de 9 años son comparables a los dados a conocer por Gianetta $^{9}$, que da cuenta de una recidiva del 1,3\% utilizando la técnica de Lichtenstein, con un seguimiento promedio de 4 años.

El uso de tapones para el tratamiento de las hernias recidivadas ha sido propuesto con entusiasmo por Shulman ${ }^{35}$, quien en 1.400 pacientes con un $91 \%$ de seguimiento da cuenta de $1,2 \%$ de re recurrencias. En nuestra serie no hubo recidivas en 9 pacientes. Nosotros hemos utilizado tapones sólo en las hernias recurrentes consecutivas a las hernioplastías con técnica de Lichtenstein y no hemos observado recurrencias.

En el presente estudio hemos querido resaltar la factibilidad de la corrección de las hernias recidivadas con anestesia local y en forma ambulatoria. En Chile, como en el resto del mundo, esta forma de proceder se da más en los centros de cirugía mayor ambulatoria, pero resalta que aún en estos centros se prefiere intervenir a los enfermos con anestesia espinal. En estas cirugías más complejas es conveniente que el cirujano tenga experiencia en el uso de la anestesia local ya que ello se vincula con una disección óptima y una correcta toma de decisiones. La falta de experiencia se asocia con una mayor tasa de recidivas ${ }^{36}$ y los mejores resultados en la cirugía con anestesia local se obtienen cuando el cirujano tiene experiencia en su aplicación ${ }^{8,11,13}$.

El conocimiento de la anatomía y, el uso de una correcta técnica disectiva y el empleo de las técnicas de reparación ciñéndose a las normas, hacen recomendable que las hernias recurrentes sean tratadas en centros con experiencia en cirugía herniaria.

Por no ser realizables con anestesia local y en forma ambulatoria, no nos hemos referido a las otras numerosas técnicas descritas para el tratamiento de las hernias inguinales recidivadas. Se ha utilizado la vía preperitoneal para instalar una malla utilizando la vía abierta de abordaje o la vía laparoscópica ${ }^{37-39}$. Las tasas de recidivas con estas 
técnicas oscilan entre $0 \%$ y $3 \%$. Ambas técnicas tienen una curva de aprendizaje prolongada que, en nuestra opinión, las aleja del campo de acción del cirujano general y deben ser realizadas con anestesia general o espinal.

\section{Conclusiones}

La cirugía ambulatoria con anestesia local, por vía anterior, aparece como una alternativa a considerar en la reparación de las hernias inguinales electivas recidivadas. El uso de material protésico es la técnica a utilizar. Ello ha contribuido a disminuir las recidivas. Cuando la PPCI se aprecia indemne, en anillos herniarios menores de $3 \mathrm{~cm}$, las técnicas fasciales, ocupan aún un espacio en el tratamiento de estos pacientes. La complejidad apreciada en el abordaje y en la reparación de estas hernias hace recomendable que ellas sean realizadas por cirujanos expertos.

\section{Referencias}

1. Bendavid R. New techniques in hernia repair. World $\mathbf{J}$ Surg. 1989;13:522-31.

2. Bendavid R. The Shouldice repair. En Nyhus LM, CDondon RE (eds) Hernia IV. Lippincot, Philadelphia, 1995, pp 223.

3. Schumpelick V. Hernien. Stuttgart: Georg Thime Verlag, 2000;394-416.

4. Deysine M. Inguinal herniorrhaphy. Reduced morbidity by service standardization. Arch Surg. 1991;126:62830.

5. Deysine M. Hernia clinic in a teaching institution. Creation and developments. Hernia 2001;5:65-9.

6. Muschaweck U. How to treat recurrent inguinal hernia. En Schumpelick V, Fitzgibbons RJ (Eds) Recurrent hernia. Berlin: Springer Verlag. 2007;289-92.

7. Schumpelick V. Discussion. En Schumpelick V, Fitzgibbons RJ (Eds) Recurrent hernia. Berlin: Springer, 2007, pp 292.

8. Kurzer M, Kark AE. Open mesh repair. En Schumpelick V, Fitzgibbons RJ (Eds) Recurrent hernia. Berlin, Springer. 2007; 292.

9. Gianetta E, Cuneo S, Vitale B, Camerini G, Marini P, Stell M. Anterior tension-free repair of recurrent inguinal hernia under local anesthesia: a 7-year experience in a teaching hospital. Ann Surg. 2000;231:132-6.

10. Acevedo A, Gallego A. Cirugía mayor ambulatoria de las hernias. Experiencia de 5 años en el CRS Cordillera Oriente de la ciudad de Santiago. Rev Chil Cir. 2004;56:166-71.

11. Acevedo A, Lombardi J. Cirugía de las hernias con anestesia local. Rev Chil Cir. 2003;56:520-6.
12. Acevedo A, Gallego A, Arriagada E, Altamirano J, Ebensperger M. Hernia inguinal recidivada. Tratamiento quirúrgico mediante la aponeurosis (arco) del músculo transverso. Rev Chil Cir. 1994;46:287-93.

13. Amid PK, Shulman AG, Lichtenstein IL. Critical scrutiny of the open "tension-free" hernioplasty. Am J Surg. 1993;165:369-71.

14. Gilbert A. An anatomic and functional classification for the diagnosis and treatment of inguinal hernia. Am J Surg. 1989;157:331-3.

15. Acevedo A, Gallegos A, Viterbo A. Fluxión funiculotesticular, una complicación frecuente en la herniorrafia inguinal. Rev Chil Cir. 2007;59:55-60.

16. Bay-Nielsen M, Kehlet H. How to create a recurrence? En Schumpelick V, Fitzgibbons RJ Ed. Recurrent hernia. Berlin: Springer, 2007;255-7.

17. Haapaniemi A, Nordin P. Present rate of failure rates (Clinical studies and epidemiological database, shortand long-term). En Schumpelick V, Fitzgibbons RJ, Ed. Recurrent hernia. Berlin, Springer. 2007;3-8.

18. Arvidsson D, Berndsen FH, Larsson LG, Leichonmarck CD, Rimbäck G, Rudberg C, et al. Randomized clinical trial comparing 5-year recurrence rate after laparoscopic versus Shouldice repair of primary inguinal hernia. Brit J Surg. 2005;92:1085-109.

19. van Veen RM, Wijsmuller AR, Vrijsland WW, Hop WC, Lange JF, Jeekel J. Long-term follow-up of a Randomized clinical trial of non-mesh versus mesh repair of primary inguinal hernia. Brit J Surg. 2007;94:506-51.

20. Bisgaard T, Bay-Nielsen M, Christensen IJ, Kehlet J. Risk of recurrence 5 years or more after primary Lichtenstein and sutured inguinal hernia repair. Br J Surg. 2007;94:1038-40.

21. EU Hernia Trialists Collaboration. Mesh compared with non-mesh methods of open groin hernia repair: Systematic review of randomized controlled trials. Br J Surg. 2000;878:854-9.

22. Porrero JL Sánchez-Cabezudo C, San Juan Benito A, López A, Hidalgo M. La herniorrafia de Shouldice en el tratamiento de la hernia inguinal primaria. Estudio prospectivo sobre 775 pacientes. Cir Esp. 2003;74:3303.

23. Millat B. Federation de recherche en chirurgie. Inguinal hernia repair. A randomized multicentric study comparing laparoscopic and open surgical repair. J Chir. 2007;144:119-24.

24. McGuire CI, Balgrie RJ, Theunissen D, Fernández NL, Chapmen LR. Outcome of laparoscopic hernia repair in South Africa private practice setting. S Afr J Surg. 2012;50:115-8.

25. Neumayer L, Giobbie-Hurder A, Johansson O, Fitzgibbons R, Dunlop D, Gibbs J, et al. Open mesh versus laparoscopic mesh repair of inguinal hernia. $\mathrm{N}$ Engl $\mathrm{J}$ Med. 2004;350:1819-27.

26. Hallén M, Bergenfelz A, Westerdahl J. Laparoscopic 
extraperitoneal inguinal hernia repair versus open mesh repair: long-term follow-up of a randomized controlled trial. Surgery 2008;143:313-7.

27. Feliú X, Torres G, Viñas X, Martínez-Rodenas M, Fernández-Sallent E, Pie J. Preperitoneal repair for recurrent inguinal hernias: Laparoscopic and open approach. Hernia 2004;8:113-6.

28. Eklund A, Rudberg C, Leijonmarck CE, Rasmussen I, Spangen L, Wickbom G, et al. Recurrent inguinal hernia: Randomized multicentric trial comparing laparoscopic and Lichtenstein Repair. Surg Endosc 2007;21:634-40.

29. Bisgaard T, Bay-Nielsen M, Kehlet H. Re-recurrence after operation for recurrent inguinal hernia. A nationwide 8 year follow-up study on the role of type of repair. Ann Surg. 2008;247:707-11.

30. Dedemadi G, Sgourakis G, Karaliotas C, Christofides T, Kouraklis G. Comparison of laparoscopic and tensionfree repair of recurrent inguinal hernias: a prospective randomized study. Surg Endosc. 2006;20:1099-104.

31. Janu PG, Sellers KD, Mangiante EC, Fielder RJ, Mason M. Recurrent inguinal hernia: preferred operative approach. Amer Surgeon 1998;64:569-74.

32. Manzanet Andrés G, Marcote Valdivieso E, Adell Crceller R, Torner Pardo A, Canales López M, Ginjer
Nogueras M. Reparación de la hernia inguinocrural mediante la técnica de Lichtenstein. Cir Esp. 1999;66:1324.

33. Haapaniemi S, Gunnarsson U, Noordin P, Nilsson E. Reoperation after recurrent groin hernia repair. Ann Surg. 2001;234:122-6.

34. Ijzermans JM, de Wilt H, Hop WJ, Jeekel H. Recurrent inguinal hernia treated by classical hernioplasty. Arch Surg. 1991;126:1097-100.

35. Shulman AG, Amid PK, Lichtenstein IL. The plug repair of 1402 recurrent inguinal hernias. 20 Year's experience. Arch Surg. 1990;125:265-7.

36. Kingsnorth AN, Britton BJ, Morris PJ. Recurrent inguinal hernia after local anesthetic repair. Brit J Surg. 1981;68:273-5.

37. Alani A, Duffy F, O’Dwyer PJ. Laparoscopic or open preperitoneal repair in the management of recurrent hernia. Hernia 1007;10:156-8.

38. Kurzer M, Belsham PA, Kark AE. Prospective study of preperitoneal mesh repair for recurrent inguinal hernia. Brit J Surg 2002;89:90-3.

39. Beets GL, Dirkden CD, Go PMNYH, Geisler FEA, Baeten CGMI, Kootstra G. Open and Laparoscopic preperitoneal mesh repair for recurrent inguinal hernia? Surg Endosc. 1999;13:323-7. 